\title{
Neural Suppression of Irrelevant Information Underlies Optimal Working Memory Performance
}

\author{
Theodore P. Zanto and Adam Gazzaley \\ Department of Neurology and Physiology, Keck Center for Integrative Neuroscience, University of California, San Francisco, San Francisco, California, \\ 94158
}

Our ability to focus attention on task-relevant information and ignore distractions is reflected by differential enhancement and suppression of neural activity in sensory cortex (i.e., top-down modulation). Such selective, goal-directed modulation of activity may be intimately related to memory, such that the focus of attention biases the likelihood of successfully maintaining relevant information by limiting interference from irrelevant stimuli. Despite recent studies elucidating the mechanistic overlap between attention and memory, the relationship between top-down modulation of visual processing during working memory (WM) encoding, and subsequent recognition performance has not yet been established. Here, we provide neurophysiological evidence in healthy, young adults that top-down modulation of early visual processing ( $<200 \mathrm{~ms}$ from stimulus onset) is intimately related to subsequent WM performance, such that the likelihood of successfully remembering relevant information is associated with limiting interference from irrelevant stimuli. The consequences of a failure to ignore distractors on recognition performance was replicated for two types of feature-based memory, motion direction and color. Moreover, attention to irrelevant stimuli was reflected neurally during the WM maintenance period as an increased memory load. These results suggest that neural enhancement of relevant information is not the primary determinant of high-level performance, but rather optimal WM performance is dependent on effectively filtering irrelevant information through neural suppression to prevent overloading a limited memory capacity.

\section{Introduction}

Both exogenously and endogenously generated attentional allocation influence how we perceive our environment. Endogenous attention, or top-down control, is driven by factors such as expectations and goals, and allows us to successfully perform higher cognitive functions (Frith, 2001; Corbetta and Shulman, 2002). Such goal-directed control is manifested as enhancing neural activity to relevant information and suppressing neural responses to irrelevant information in sensory cortical regions, a process known as top-down modulation (Fuster, 1990; Duncan et al., 1997; Gazzaley et al., 2005b).

The neural biasing afforded by top-down modulation is thought to serve memory by favoring the effective encoding of relevant information (Rainer et al., 1998; Ploner et al., 2001). It has been suggested that the contents of working memory (WM) can be conceptualized as active internal representations maintained within the focus of attention (Cowan, 1995). Neuroimaging studies have supported this by identifying a mechanistic overlap between attention and WM (LaBar et al., 1999; Awh and Jonides, 2001). However, the nature of this interaction may depend on the specific aspects of attention and memory processes

\footnotetext{
Received Sept. 25, 2008; revised Jan. 23, 2009; accepted Jan. 26, 2009.

This work was supported by National Institutes of Health Grants K08-AG025221 and R01-AG30395 and the Ellison Medical Foundation. We thank Kelly Hennigan for her assistance in data collection.

Correspondence should be addressed to Adam Gazzaley, Department of Neurology and Physiology, Keck Center for Integrative Neuroscience, University of California, San Francisco, MC2240, 600 16th Street, Room N472J, San Francisco, CA 94158. E-mail: adam.gazzaley@ucsf.edu.

D01:10.1523/JNEUROSCI.4621-08.2009

Copyright $\odot 2009$ Society for Neuroscience $\quad$ 0270-6474/09/293059-08\$15.00/0
}

being considered (Awh et al., 2006). Here, we explore the relationship between selective attentional modulation during WM encoding and subsequent recognition performance.

Due to overlapping neural substrates, competition for shared resources between visual selective attention and WM encoding demands may limit processing capabilities (Mayer et al., 2007). Load theory predicts a WM load will produce an impairment in selective attention marked by increased distractibility (Lavie et al., 2004). In addition, the presence of distractors have been shown to impair WM performance (Eriksen and Eriksen, 1974), presumably due to irrelevant information overloading a limited memory capacity (Vogel et al., 2005). However, it is unknown whether distraction negatively impacts subsequent WM performance via reduced neural enhancement for relevant information, suppression for irrelevant information or a combination of both. To address this issue, we dissociate neural signatures of enhancement and suppression to relevant and irrelevant information, respectively. We hypothesize that optimal WM performance may be achieved only although efficient filtering of irrelevant information to prevent overloading limited memory stores.

Electroencephalography (EEG) was recorded while participants were presented four sequential apertures of dots: two were differently colored and stationary, two were gray and moving coherently in different directions (see Fig. 1). Attention was selectively modulated by instructing participants to remember the "color" hues (ignore motion; two item WM task), remember the "motion" direction (ignore color; two item WM task), or "remember both" colors and directions of motion (four item WM task). WM was tested after a 4-s delay with a probe stimulus. 


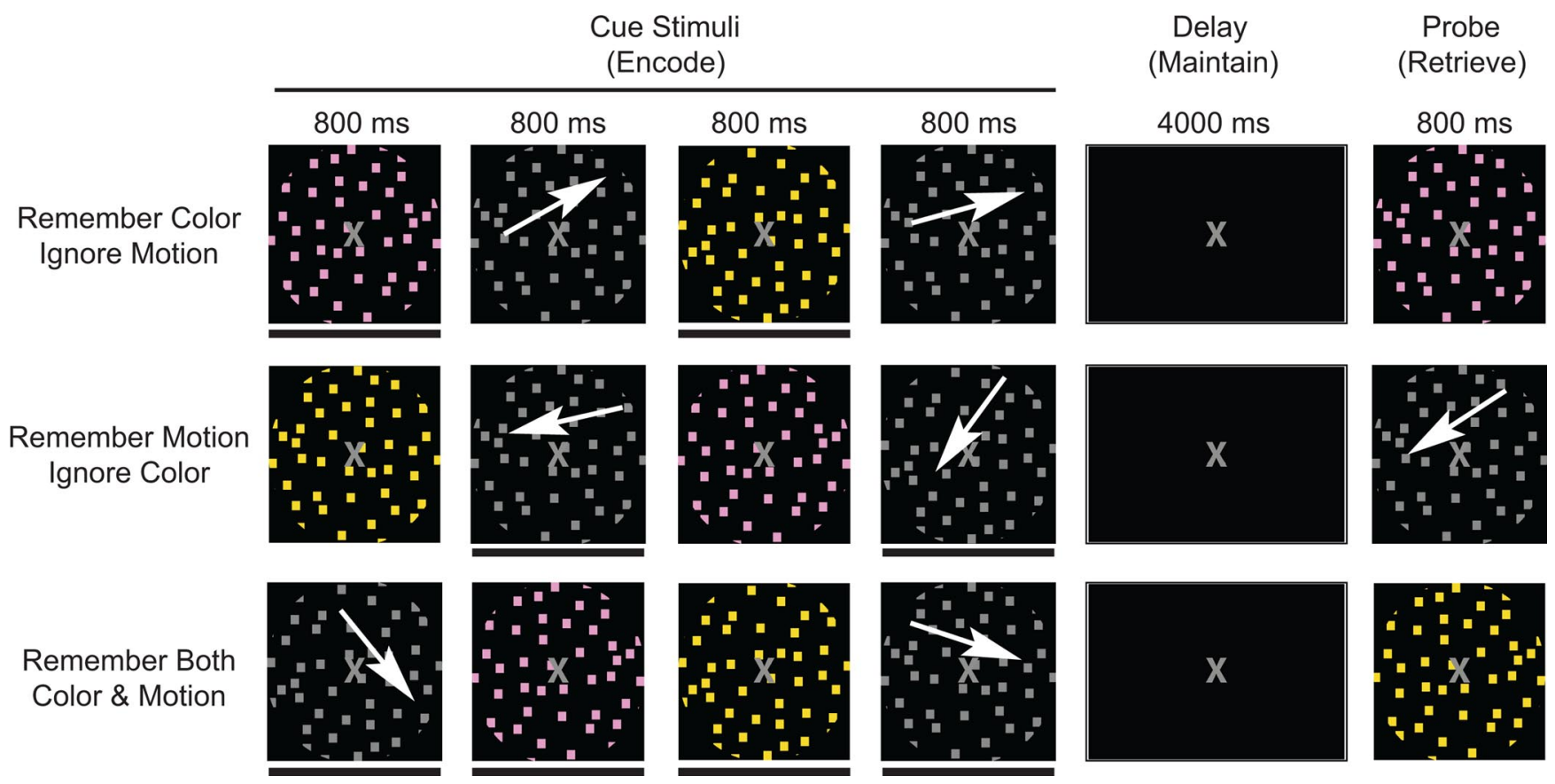

Figure 1. Experimental paradigm. White arrows indicate motion and were not present during the experiment. A black bar below a cue stimulus (also not present during the experiment) indicates it is an item to be remembered. The pictures approximate stimuli appearance (see Materials and Methods for details).

Participants were instructed to indicate whether the probe matched one of the relevant items held in memory. This design allowed us to examine neural measures during distinct cognitive stages of the tasks (i.e., WM encoding, maintenance and retrieval). Moreover, the temporal separation of relevant and irrelevant stimuli allowed us to dissociate the impact of attending and ignoring on WM performance. To yield more sensitive indicators of performance, participants were instructed to respond as quickly as possible, without sacrificing accuracy.

\section{Materials and Methods}

Participants. Twenty healthy individuals (mean age 25.1 years; range 20-33 years; 11 males) gave informed consent to participate in the study according to procedures approved by the University of California. All participants had normal or corrected to normal vision. One participant's data was not included in the analysis due to a low signal-to-noise ratio, which prevented ERP peak identification.

Stimuli. The stimuli consisted of a circular aperture of 290 dots $\left(0.08^{\circ}\right.$ $\times 0.08^{\circ}$ each) that subtended $8^{\circ}$ of visual angle at a $75 \mathrm{~cm}$ viewing distance and were centered at the fovea. Two types of dots were used during the experiment: (1) gray and moving coherently at $10^{\circ}$ per second or (2) stationary and colored along the tritan axis. All colored and gray dots were equated for brightness by minimizing heterochromatic flicker in tests performed before the experiment for each subject (Hardy et al., 2005). Stimuli were presented with a gray fixation cross in the center of the circular aperture on a black background $\left(0.32 \mathrm{~cm} / \mathrm{m}^{2}\right)$.

Thresholding and experimental procedure. After all stimuli were equated for brightness, participants went through two thresholding tests (one for motion, one for color) to minimize discriminability differences between participants. A staircase procedure required participants to determine whether two stimuli (directions of motion or colors) were different from each other. The two stimuli were presented for $800 \mathrm{~ms}$ each and separated by $2000 \mathrm{~ms}$. The procedure continued until a "just 100\%" level of performance was reached, meaning if the stimuli were any more similar, performance would drop below $100 \%$. Thresholding determined the 12 possible directions of motion (three per quadrant, cardinal axes excluded) and the six possible colors each participant received during the experiment.

One experimental trial is depicted in Figure 1 for each condition.
Participants were presented with 60 trials per condition over the course of the experiment. The stimuli for each trial were randomly selected from the predetermined sets of stimuli, constraining the directions of motion to one quadrant. An $800 \mathrm{~ms}$ interstimulus-interval contains stationary gray dots as well as the $1000 \mathrm{~ms}$ before the first cue stimulus. During the motion and color conditions, $50 \%$ of the probe stimuli matched a previously presented stimulus whereas the other $50 \%$ was not presented during the trial. Moreover, probe stimuli always consisted of the to-beremembered category (i.e., motion probes during the motion condition, color probes during the color condition). A passive viewing condition was conducted in addition to the three displayed in Figure 1, but was not included in the current analyses.

Electrophysiological recordings. Electrophysiological signals were recorded at $1024 \mathrm{~Hz}$ through a 24-bit BioSemi ActiveTwo 64-channel Ag$\mathrm{AgCl}$ active electrode EEG acquisition system (Cortech Solutions, LLC). Electrode offsets were maintained between $+/-20 \mathrm{mV}$. Raw EEG data were referenced to the average off-line. Eye artifacts were removed through an independent component analysis by excluding components consistent with topographies for blinks and eye movements and the electrooculogram time-series. One-second epochs were extracted from the data beginning $200 \mathrm{~ms}$ prestimulus onset and ending $800 \mathrm{~ms}$ poststimulus onset. This preprocessing was conducted in Brain Vision Analyzer (Cortech Solutions) and exported to Matlab (The Mathworks) for all subsequent analyses. Each trial contained four epochs, two attended and two ignored, whereas all four epochs were attended in the remember both condition. Thus, over the 60 trials, 120 epochs were acquired for each event-related potential (ERP) of interest. Epochs were bandpass filtered from 1 to $30 \mathrm{~Hz}$ and those that exceeded a voltage threshold of $+/-50 \mu \mathrm{V}$ were rejected. A $200 \mathrm{~ms}$ prestimulus baseline was subtracted from each epoch before calculating the ERP. Peak positive deflection (P1) values were chosen as the largest amplitude between 50 and $150 \mathrm{~ms}$, whereas the negative deflections (N1) were identified as the most negative amplitude between 120 and $220 \mathrm{~ms}$.

Mean ERP amplitudes (+/- $5 \mathrm{~ms})$ were compared through repeated measures ANOVAs with a Geisser-Greenhouse correction (when appropriate) from electrodes of interest (P1: PO8 for motion, IZ for color; N1: P10 for motion, IZ for color). These electrodes of interest were chosen to be the largest ERP from the grand average when collapsed across participants and conditions. Post hoc analysis used paired $t$ tests with a false- 
A
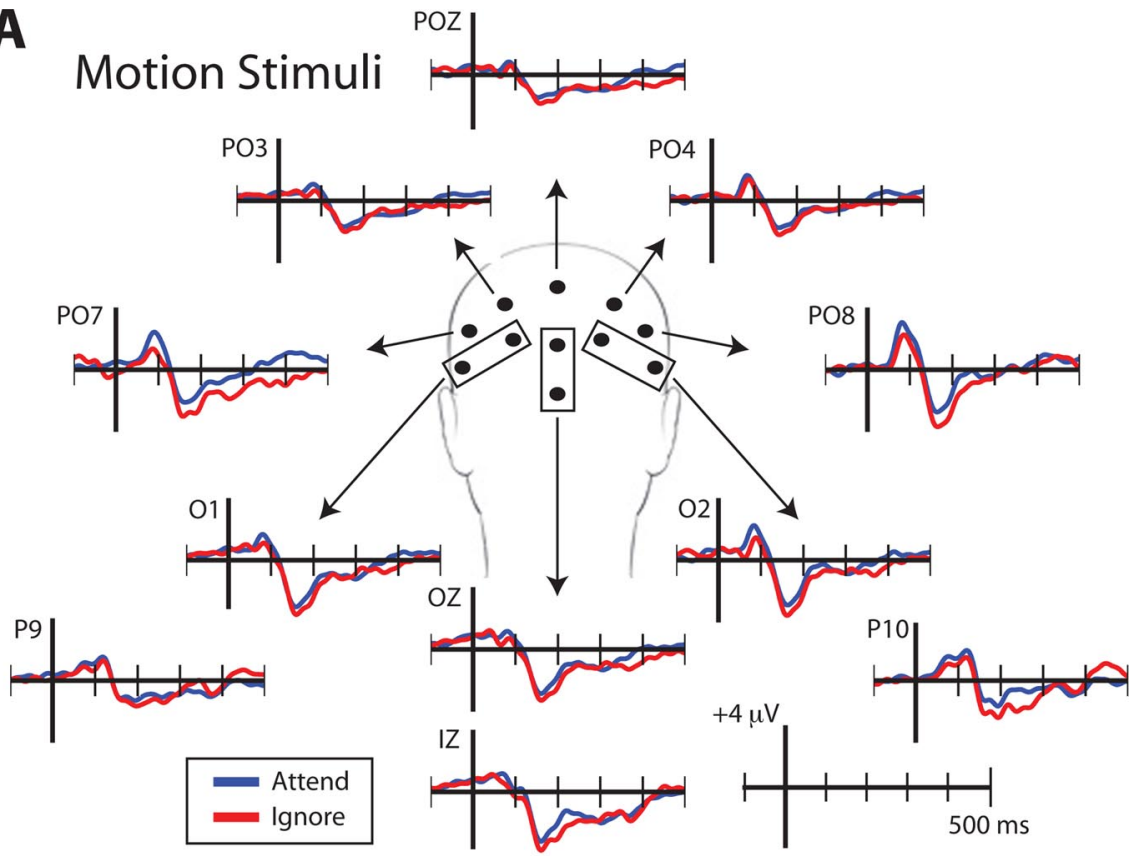

B
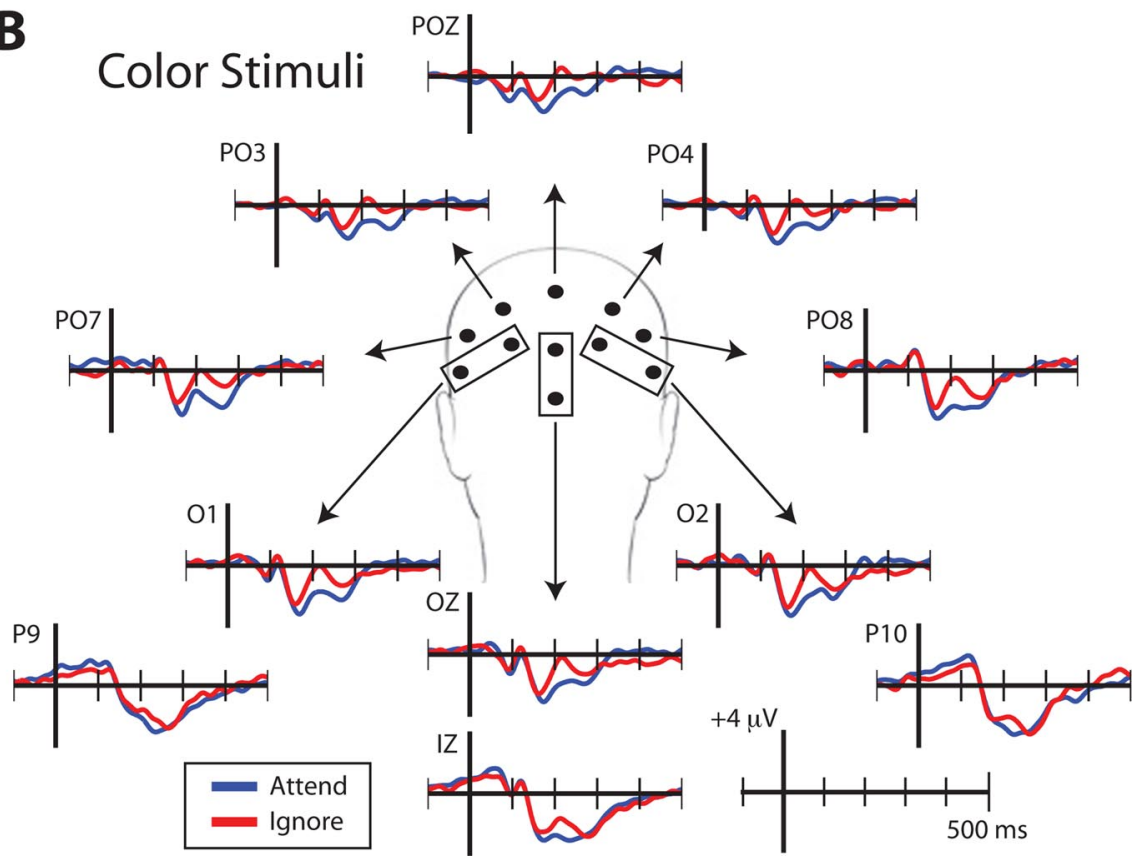

Figure 2. ERP time-series from select posterior-occipital electrodes when viewing, motion $(\boldsymbol{A})$ or color stimuli $(\boldsymbol{B})$. Note: Electrode locations are an approximation.

discovery rate correction for multiple comparisons (Benjamini and Hochberg, 1995). Data from the delay period was subject to the same artifact rejection procedure as for the ERP analysis; however, the voltage threshold was set to $+/-100 \mu \mathrm{V}$, and the delay period data was not bandpass filtered before the wavelet convolution to resolve frequencies from 4 to $70 \mathrm{~Hz}$. The time-frequency decomposition of the delay period was acquired via complex Morlet wavelets (family ratio: $f_{\mathrm{o}} / \sigma_{\mathrm{f}}=8$ ) as implemented through EEGLAB (Delorme and Makeig, 2004). Induced activity was acquired by calculating time-frequency maps for each epoch and averaging over all maps, as detailed in Tallon-Baudry et al. (1998). Each frequency was normalized over time with a $z$-score across all conditions per participant. Mean time-frequency $z$-scores from five electrodes of interest were subjected to ANOVAs with a Geisser-Greenhouse correction when appropriate. Electrodes of interest were selected to represent frontal, parietal and occipital regions (FCZ, PZ, P5, P6, and IZ).
Post hoc analysis used paired $t$ tests and a falsediscovery rate correction for multiple comparisons.

\section{Results}

\section{Behavior}

Behavioral analysis showed WM response time (RT) to increase as memory load increased (two items: motion $=908 \mathrm{~ms}$, $\mathrm{SEM}=58 \mathrm{~ms}$, color $=906 \mathrm{~ms}, \mathrm{SEM}=64$ ms; four items: remember both $=1019$ $\mathrm{ms}, \mathrm{SEM}=60 \mathrm{~ms} ; p<0.005)$. There was no significant difference between color and motion tasks in RT ( $p>0.05)$, and no significant differences between any of the tasks in accuracy (motion $=78 \%$, SEM $=$ $2 \%$; color $=74 \%$, SEM $=2 \%$; remember both $=73 \%$, SEM $=2 \%$; $p>0.05)$.

To evaluate the relationship between neural measures associated with top-down modulation (attending and ignoring) and indicators of WM performance, trials from the motion and color tasks for each participant were split by fast and slow responses across the median RT. This approach is consistent with many other studies that have used RT as a sensitive indicator of behavioral performance (Stroop, 1935; Posner et al., 1978). Indeed, fast RT trials were associated with enhanced WM accuracy in both motion (fast $=82 \%$, SEM $=3 \%$; slow $=73 \%$, $\mathrm{SEM}=3 \% ; p<0.005)$ and color tasks (fast $=81 \%$, SEM $=2 \%$; slow $=67 \%$, $\mathrm{SEM}=2 \% ; p<0.001)$. Subsequent analyses of ERP data are separately evaluated for all trials (average performance), highperformance trials (i.e., fast RT) and lowperformance trials (i.e., slow RT).

\section{Event-related potentials during stimulus encoding}

To identify neural markers of top-down modulation during the encoding period of each task, we compared peak amplitude measures of ERP waveforms time-locked to relevant and irrelevant stimuli during the two-item WM tasks. This analysis focused on two peaks, the $\mathrm{P} 1$ ( $\sim 100 \mathrm{~ms}$ poststimulus onset) and the $\mathrm{N} 1(\sim 170 \mathrm{~ms}$ poststimulus onset), which reflect early stages of visual processing and have previously been shown to be modulated by different types of attention (Rugg et al., 1987; Hillyard et al., 1998; ValdesSosa et al., 1998). Figure 2 displays ERPs to attended and ignored stimuli from posterior electrodes. Attentional modulation at the $\mathrm{P} 1$ and N1 is apparent in parietal and occipital regions. Therefore, average peak amplitude measures from the electrodes of interest for the P1 and N1 were submitted to an ANOVA with stimulus (color/motion) and condition (attend/ignore) as factors.

The P1 amplitude displayed a main effect for both stimulus $\left(F_{(1,18)}=13.74, p<0.005\right)$ and condition $\left(F_{(1,18)}=6.08, p<\right.$ $0.05)$, indicating motion yields a larger $\mathrm{P} 1$ than color (motion: $3.21 \mu \mathrm{V}, \mathrm{SEM}=0.30 \mu \mathrm{V}$; color: $1.83 \mu \mathrm{V}, \mathrm{SEM}=0.15 \mu \mathrm{V})$ and 
that the amplitude of the P1 was modulated by attention (attend $=2.72 \mu \mathrm{V}$, SEM $=0.29 \mu \mathrm{V}$; ignore $=2.32 \mu \mathrm{V}$, SEM $=0.23 \mu \mathrm{V})$. Additionally, a stimulus by condition interaction $\left(F_{(1,18)}=9.42\right.$, $p<0.01$ ) was observed. Post hoc analysis identified significant attentional modulation (attend $>$ ignore) of the P1 amplitude for motion stimuli (Fig. $3 a)(p<0.01)$, while no such modulation was observed for color $(p>0.05)$. Together, these results indicate that the P1 elicited by motion stimuli is modulated by attention.

The N1 amplitude displayed a main effect for condition $\left(F_{(1,18)}=8.57, p<\right.$ 0.01 ), such that attending to the stimuli elicits a larger (i.e., more negative) amplitude than ignoring the stimuli (attend $=$ $-4.90 \mu \mathrm{V}, \mathrm{SEM}=0.41 \mu \mathrm{V}$; ignore $=$ $-4.51 \mu \mathrm{V}, \mathrm{SEM}=0.42 \mu \mathrm{V})$. Moreover, $\mathrm{a}$ stimulus by condition interaction $\left(F_{(1,18)}\right.$ $=15.50 ; p<0.005)$ was also observed. Post hoc analysis indicated that the N1 amplitude to color stimuli was modulated by attention (Fig. 3c) $(p<0.001)$, whereas motion stimuli did not display attentional modulation ( $p>0.05)$. Thus, the $\mathrm{N} 1$ elicited by color stimuli is modulated by attention and the color amplitude drove the observed main effect.

Overall, the P1 amplitude to motion stimuli was modulated by attention whereas the N1 to color stimuli displayed attentional modulation. Therefore, further ERP analysis used these measures as functional markers of top-down modulation during WM encoding.

To identify if there as an impact of WM load on markers of early attentional modulation, paired $t$ tests were conducted to compare the remember both (four-item) task with the two-item tasks (P1 for motion and N1 for color). Results indicate that stimuli from the four-item task elicited similar ERPs as stimuli from the two-item tasks for the same relevant dimension (P1 for motion and N1 for color; $p>0.05$ ).

To evaluate if the degree of top-down modulation differed across low- and high-performance trials in the two-item tasks, independent evaluations of these trial subtypes were conducted. Comparable with the behavioral analysis, high- and lowperformance ERPs were calculated based on the median RT split from all artifact-free trials. Peak P1 and N1 amplitudes from motion and color stimuli, respectively, were submitted to an ANOVA with condition (attend/ignore) and performance (high/ low) as factors.

The $\mathrm{P} 1$ evoked by motion stimuli yielded main effects for both condition $\left(F_{(1,18)}=9.25, p<0.01\right)$ and performance $\left(F_{(1,18)}=\right.$ $7.09, p<0.05)$, such that attentional modulation (attend $>$ ignore) was observed (attend $=3.56 \mu \mathrm{V}, \mathrm{SEM}=0.32 \mu \mathrm{V}$; ignore $=$ $2.78 \mu \mathrm{V}, \mathrm{SEM}=0.32 \mu \mathrm{V})$ and low-performance trials produced a larger P1 amplitude compared with high-performance trials $($ low $=3.42 \mu \mathrm{V}, \mathrm{SEM}=0.31 \mu \mathrm{V}$; high $=2.92 \mu \mathrm{V}, \mathrm{SEM}=0.34$ $\mu \mathrm{V})$. Additionally, a condition by performance interaction was observed $\left(F_{(1,18)}=4.81, p<0.05\right)$. Post hoc analysis revealed significant modulation (attend $>$ ignore) during highperformance trials (Fig. $3 b$, light gray bars) $(p<0.005)$, but not during low-performance trials (Fig. $3 b$, dark gray bars) $(p>$ 0.05). Furthermore, paired $t$ tests on the magnitude of modulation (attend - ignore) identified significantly reduced attentional modulation in low-performance relative to highperformance trials $(p<0.05)$. Therefore, high WM performance for motion stimuli is associated with $\mathrm{P} 1$ attentional modulation, whereas a lack of modulation at the P1 is associated with low WM performance.

The N1 evoked by color stimuli displayed a main effect for condition $\left(F_{(1,18)}=7.61, p<0.05\right)$, such that attentional modulation (attend $>$ ignore, i.e., more negative) was observed (attend $=-4.58 \mu \mathrm{V}, \mathrm{SEM}=0.41 \mu \mathrm{V}$; ignore $=-3.77 \mu \mathrm{V}, \mathrm{SEM}=$ $0.36 \mu \mathrm{V})$. Moreover, a condition by performance interaction was observed $\left(F_{(1,18)}=5.21, p<0.05\right)$. Similar to the P1 for motion stimuli, post hoc analysis of the color-evoked N1 amplitude revealed significant modulation (attend $>$ ignore) during highperformance trials (Fig. $3 d$, light gray bars) ( $p<0.005$ ), but not during low-performance trials (Fig. $3 d$, dark gray bars) $(p>$ $0.05)$. Furthermore, paired $t$ tests on the magnitude of modulation (attend - ignore) identified significantly reduced attentional modulation in low-performance relative to highperformance trials $(p<0.05)$. Thus, high WM performance for color stimuli is associated with $\mathrm{N} 1$ attentional modulation, whereas a lack of modulation at the $\mathrm{N} 1$ is associated with low WM performance.

These results suggest that top-down modulation of the P1 for motion stimuli and the N1 for color stimuli reflects subsequent WM performance after the delay period. However, this analysis did not identify whether the absence of modulation observed 


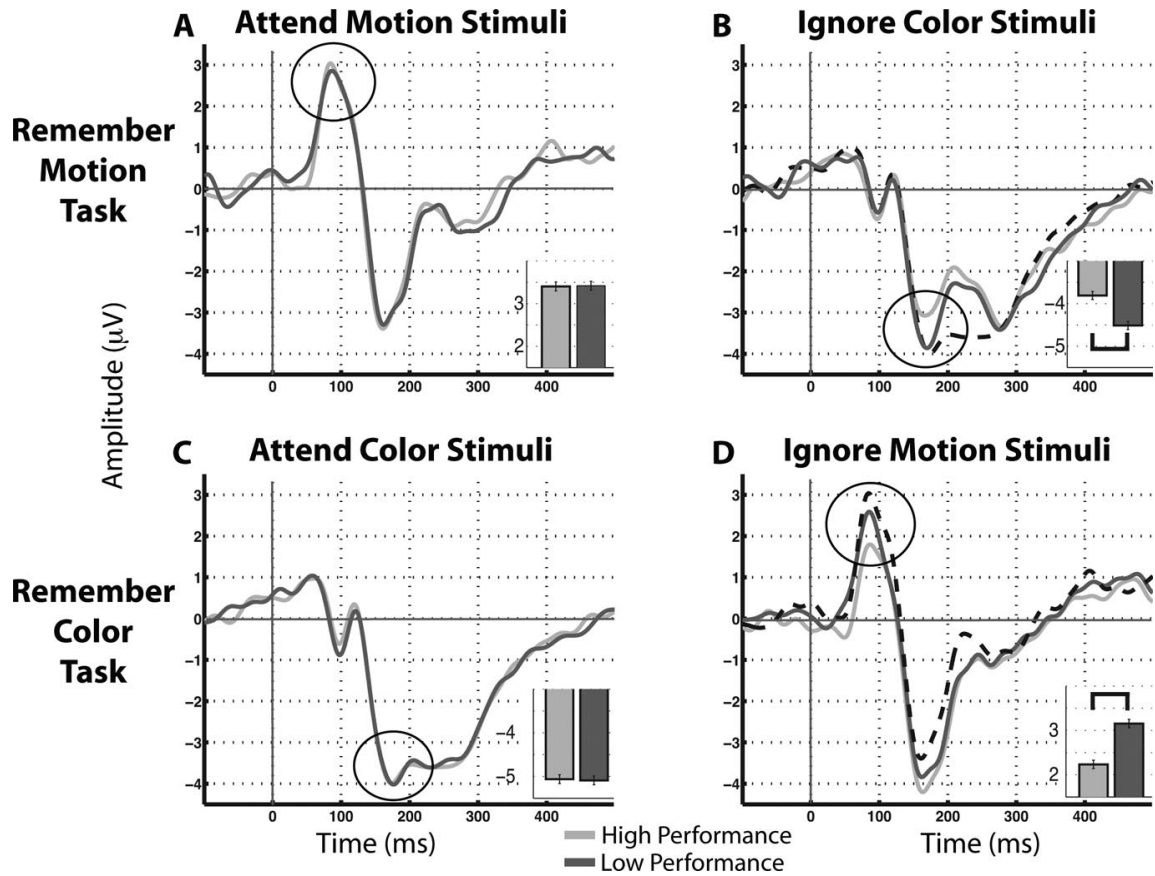

Figure 4. ERP comparisons of low-performance (dark gray line) and high-performance (light gray line) trials for attended and ignored stimuli. Inset, Bar graphs compare designated peak ERP measures between low- and high-performance trials. $\boldsymbol{A}$, No differences observed between low- and high-performance at the P1 peak when attending to motion $(\boldsymbol{A})$ or the $\mathrm{N} 1$ peak when attending to color ( $\boldsymbol{C}$. $\boldsymbol{B}$, When participants are instructed to ignore color, an enhanced $\mathrm{N} 1$ is observed during low-performance trials, which is similar in magnitude to the $\mathrm{N} 1$ when attending to color (dashed line: waveform from C). $\boldsymbol{D}$, When instructed to ignore motion, an enhanced $\mathrm{P} 1$ is observed during low-performance trials that is similar in magnitude to the $\mathrm{P} 1$ when attending to motion (dashed line: waveform from $A$ ). Error bars reflect the SEM.

during the low-performance trials was the result of a lack of attention to relevant stimuli, failure to ignore irrelevant stimuli, or a combination of both. To assess why top-down modulation differs with performance, additional post hoc analyses on the ERPs from the low- and high-performance trials directly compared both relevant and irrelevant stimuli from the two-item tasks.

Analysis revealed a marked difference based on stimulus relevance. When participants viewed relevant stimuli, no difference existed in the ERPs between low- and high-performance trials, for either motion or color stimuli (Fig. $4 a, c)(p>0.05)$. However, when participants viewed irrelevant stimuli, the ERPs were significantly different across the performance split, such that neural markers of attentional modulation were increased in lowperformance trials (Fig. $4 b, d$, motion and color) $(p<0.05$ ). Moreover, the peak ERP amplitude to irrelevant stimuli during low-performance trials did not differ significantly from those associated with relevant stimuli during the low-, or even highperformance trials (Fig. $4 b$, $d$, dashed black line). This data suggests that during low-performance trials, participants allocated as much attention to irrelevant stimuli as they did on trials when that same stimulus was task-relevant. Together, these results indicate that WM performance is not associated with fluctuations in attention to relevant stimuli during encoding, but rather with fluctuations across trials in adequately ignoring irrelevant stimuli. This result is supported by ERP analyses comparing low- and high-performance trials from the remember both task, in which all stimuli were relevant. The analysis revealed no significant difference between the P1 for attended motion stimuli or the $\mathrm{N} 1$ for attended color stimuli across performance subtypes $(p>0.05)$, thus supporting the conclusion that variation in attention to relevant stimuli during encoding was not associated with WM performance level.

\section{Time-frequency analysis during WM maintenance}

In the previous section, we dissociated neural signatures of attending and ignoring visual stimuli during encoding and revealed their differential impact on subsequent WM performance. However, we are also poised to explore the neural consequences of improper attentional allocation during encoding on WM maintenance activity. Given that low-performance trials are associated with a failure to ignore irrelevant stimuli, we hypothesized that during these trials participants encoded more items into memory than during the highperformance trials, resulting in interference that subsequently diminished recognition performance. To evaluate this, an initial time-frequency analysis of the delay period was conducted to determine whether performance differences exist in the alpha $(8-12 \mathrm{~Hz})$, beta $(12-20 \mathrm{~Hz})$ or gamma $(20-50 \mathrm{~Hz})$ frequency ranges.

For each frequency band, average values from consecutive $500 \mathrm{~ms}$ timefrequency windows with $50 \%$ overlap were extracted across the delay period beginning $250 \mathrm{~ms}$ after the final stimulus offset to prevent the confound of including a stimulus offset response. Average values were submitted to an ANOVA with stimulus (color/motion), performance (high/low), time-window and electrode as factors. Five electrodes distributed about the scalp were selected for this analysis: FCZ, PZ, P5, P6, and IZ. Figure 5 shows desynchronized activity (decreased activity in blue) in the alpha and beta bands during the first $1500 \mathrm{~ms}$ of the delay period, whereas subsequent activity is synchronized (increased activity in red). To explore changes independently within these regions of synchronized and desynchronized activity, the alpha and beta bands were subjected to two separate ANOVAs: one with timewindows spanning 250-1500 $\mathrm{ms}$ and one with time-windows spanning 1500-4000 ms of the delay period. The gamma band displayed no such temporal structure; therefore, the analyzed time-windows spanned the entire delay period.

Desynchronized alpha activity early in the delay period displayed no main effects or interactions. However, synchronized alpha activity later in the delay period exhibited main effects for both time $\left(F_{(8,144)}=5.36, p<0.05\right)$ and electrode $\left(F_{(4,72)}=4.68\right.$, $p<0.005)$, such that the alpha activity peaks between 1750 and $2250 \mathrm{~ms}$ and was greatest at electrode P6.

Desynchronized beta activity early in the delay period displayed main effects for time $\left(F_{(3,54)}=12.98, p<0.001\right)$ and electrode $\left(F_{(4,72)}=4.14, p<0.05\right)$. The decrease in activity was greatest early in the delay period $(250-750 \mathrm{~ms})$ and was most pronounced at electrode P6. Furthermore, a performance by time interaction was observed $\left(F_{(3,54)}=6.45, p<0.005\right)$. Post hoc analysis indicated that the performance difference was greatest at the earliest time-window $(250-750 \mathrm{~ms})$ such that lowperformance trials (mean $z$-score: -0.57 , SEM $=0.04$ ) were more desynchronized than the high-performance trials (mean $z$-score: $-0.31, \mathrm{SEM}=0.04 ; p<0.005)$. Synchronized beta activity displayed main effects for time $\left(F_{(8,144)}=13.63, p<0.001\right)$, electrode $\left(F_{(4,72)}=6.73, p<0.001\right)$ and stimulus $\left(F_{(1,18)}=4.91\right.$, 


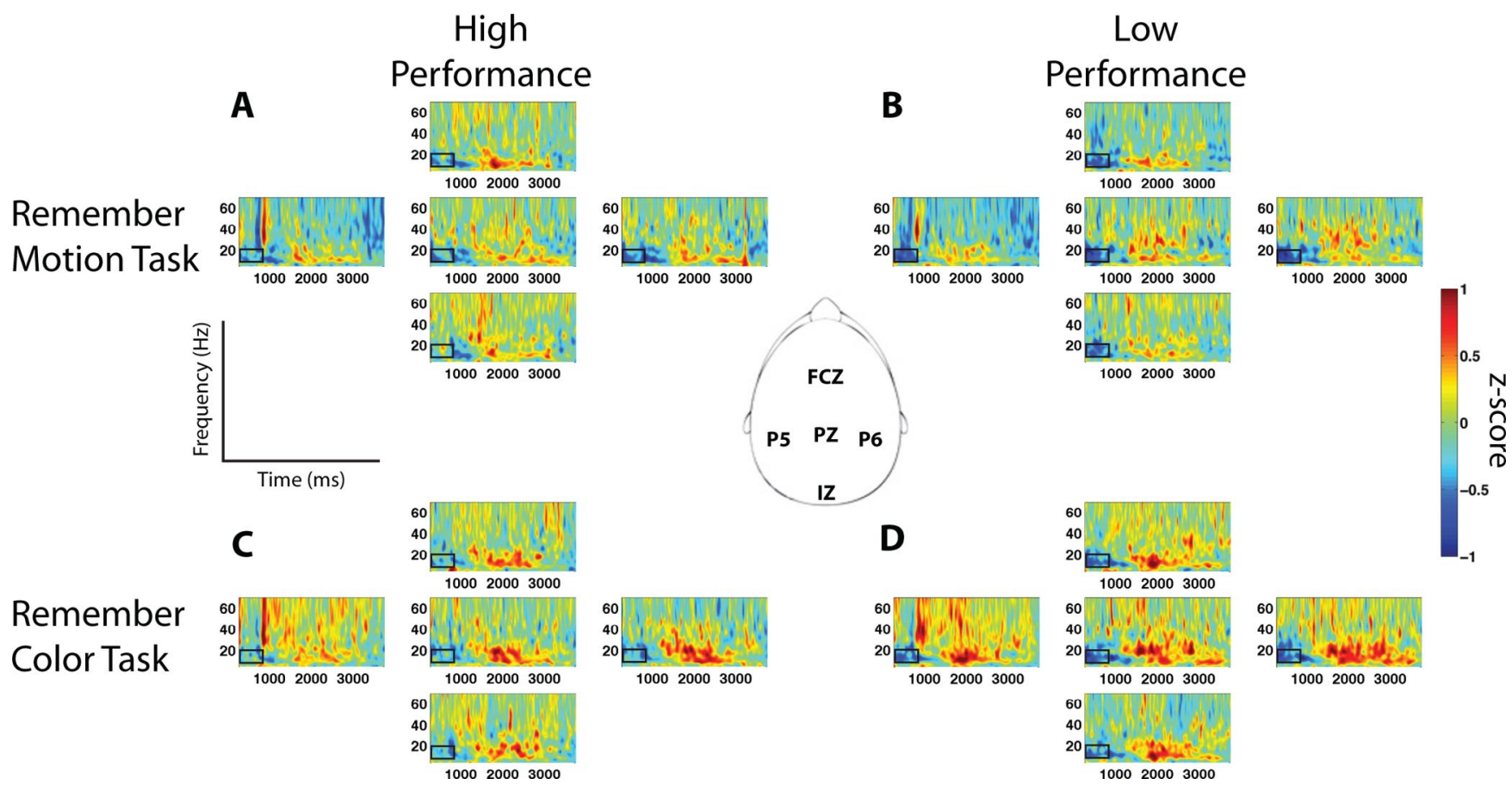

Figure 5. Time-frequency maps from five electrodes of interest during the delay period. The top maps are from the motion task during high-performance trials $(\boldsymbol{A})$ and low-performance trials $(\boldsymbol{B})$. The lower maps are from the color task during high-performance trials $(\boldsymbol{C})$ and low-performance trials $(\boldsymbol{D})$. Black boxes highlight performance differences in the beta band such that greater desynchronization is observed during low-performance trials (dark blue).

$p<0.05)$. The synchronized beta activity peaked between 1500 and $2000 \mathrm{~ms}$, was greatest at electrode P6 and was more pronounced during the delay period of the color task.

Analysis of the gamma band revealed a main effect for time $\left(F_{(13,234)}=9.86, p<0.001\right)$, such that it was most desynchronized at the beginning of the delay period and displayed peak synchronization between 1250 and $1750 \mathrm{~ms}$ into the delay period. Additionally, a time by electrode interaction $\left(F_{(52,936)}=2.91, p<\right.$ $0.005)$ was observed.

Together, these results show that performance differences only exist early in the delay period as reflected by beta-band desynchronization. To determine if low-performance trials from motion and color tasks (encode two items) were comparable with remember both (encode all four items) during the WM maintenance period, further analysis of the delay period focused on the early desynchronized beta-band activity (Fig. 5 , black boxes). Because no stimulus main effect or interaction was observed, subsequent analysis of the spectral activity during the delay period was averaged across motion and color data. Thus, comparisons between performance and WM load used an ANOVA with electrode and type (high performance two-item task, low performance two-item task and average performance four-item task) as factors.

Analysis of the desynchronized beta activity resulted in main effects for electrode $\left(F_{(4,72)}=8.47, p<0.001\right)$ and type $\left(F_{(2,36)}=4.64, p<0.05\right)$. Post hoc analysis indicated that beta desynchronization was greater at the lateral electrodes (mean $z$-scores: P5 $=-0.55, \mathrm{SEM}=0.06 ; \mathrm{P} 6=-0.60, \mathrm{SEM}=0.05$ ) compared with the midline electrodes (mean $z$-scores: $\mathrm{FCZ}=$ $-0.42, \mathrm{SEM}=0.07 ; \mathrm{PZ}=-0.41, \mathrm{SEM}=0.07 ; \mathrm{IZ}=-0.37$, SEM $=0.07 ; p<0.05)$. Furthermore, the high WM load task (Fig. 6, black bar) yields more beta desynchronization compared with the high-performing two-item task (Fig. 6, light gray bar), whereas no difference is observed between the high

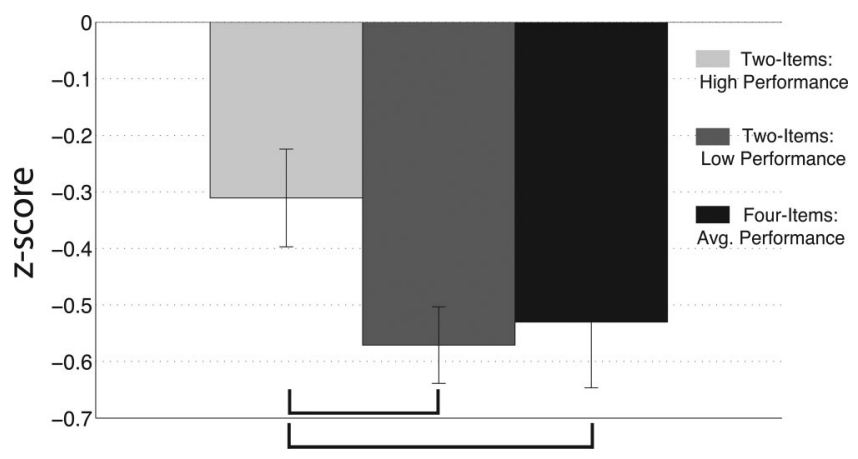

Figure 6. Average beta-band ( $12-20 \mathrm{~Hz}$ ) activity between 250 and $750 \mathrm{~ms}$ into the delay period. Greater beta desynchronization is observed during the four-item task (black bar) and low-performance two-item task (dark gray bar) relative to the high-performance two-item task (light gray bars). The black brackets indicate a significant difference at $p<0.05$. Error bars reflect the SEM.

WM load and the low-performing two-item task (Fig. 6, dark gray bar). Overall, these results suggest that attending to two relevant stimuli and failing to ignore two irrelevant stimuli (i.e., two-item tasks, low-performance) yields WM maintenance activity measures that are comparable with maintaining all four items in WM. This data is paralleled by behavioral analyses that revealed the RT of low-performance, two-item trials $($ motion $=1084 \mathrm{~ms}, \mathrm{SEM}=76 \mathrm{~ms}$; color $=1120 \mathrm{~ms}$, SEM $=94 \mathrm{~ms}$ ) were not significantly different from the mean $\mathrm{RT}$ of the four-item task (remember both $=1025 \mathrm{~ms}$, SEM = $57 \mathrm{~ms} ; p>0.05)$, whereas high-performance trials were significantly faster (motion $=713 \mathrm{~ms}$, SEM $=34 \mathrm{~ms}$; color $=692$ $\mathrm{ms}, \mathrm{SEM}=28 \mathrm{~ms}$, remember both $=1025 \mathrm{~ms}, \mathrm{SEM}=57 \mathrm{~ms}$; $p<0.05)$. Together, these data suggest that failure to ignore irrelevant information reduces WM performance by increasing the memory load during WM maintenance. 


\section{Event-related potentials during probe stimulus}

To identify if WM performance may also be influenced by attentional changes at retrieval, ERPs to the probe stimuli were analyzed. No P1 or N1 differences between low- and highperformance trials on either motion or color tasks were observed in response to the probe stimulus ( $p>0.05$, all comparisons). This suggests that variation in WM performance across trials was due to improper allocation of attention during the encoding stage and not during WM retrieval.

\section{Discussion}

The current results indicate that early components of the visual ERP, the P1 and N1, are modulated by attention to motion and color stimuli, respectively. However, significant top-down modulation was not present during low-performance trials (i.e., trials with slower response times and decreased accuracy). Further analysis revealed that the lack of significant modulation in the low-performance trials was due to improper allocation of attention exclusively to irrelevant stimuli. Although the color and motion tasks required maintenance of only two of the four items presented, spectral analysis suggested inappropriate attentional allocation to irrelevant stimuli in the low-performance trials was reflected in the delay period as an increased WM load, similar to encoding four items in memory. This differential impact of attending and ignoring information on subsequent WM maintenance and recognition performance was replicated for two types of feature-based memory (i.e., color and motion).

It is well established that neural activity associated with irrelevant information can be suppressed early in the visual processing system (for review, see Hillyard et al., 1998). Although much of this data were generated from spatial attention studies, we provide evidence that feature-based attention (i.e., color hue and motion direction) also results in modulation at early stages in visual processing, corroborating recent reports (Beer and Röder, 2004; Schoenfeld et al., 2007). We extend these findings by showing that early top-down modulation is not consistent across trials, notably for the suppression of irrelevant information.

Despite our increasing understanding of neural suppression and an extensive literature connecting attention and memory, a direct link between top-down suppression and WM performance in young adults has been elusive. Therefore, in the current study we implemented a challenging feature-based WM task and used RT measures to dissociate low- and high-performance trials from all participants to identify neural correlates of optimal WM recognition. This approach is consistent with many other studies that have used RT as a sensitive indicator to elucidate neuralperformance relationships (Rypma and D'Esposito, 2000; Womelsdorf et al., 2006; Talsma et al., 2007). Here, we reveal that low-performance trials with slower RTs were associated with spectral markers of increased memory load during maintenance (i.e., beta-band desynchronization), consistent with classic behavioral findings that a higher memory load leads to increased recognition RT (Sternberg, 1966, 1969).

An extensive behavioral literature has revealed that cognitive performance is diminished by distraction. For example, in the Flanker task perceptual performance, as indicated by the RT for a discrimination of a central stimulus, is impaired by the presence of flanking irrelevant information, thus revealing the detrimental influence of distractors on perception (Eriksen and Eriksen, 1974). EEG data has suggested that the impact of distraction on perceptual performance in the Flanker task occurs early in the visual stimulus processing stream (Scerif et al., 2006). It has also been suggested by behavioral studies that appropriate top-down allocation of attentional resources is critical for successful WM performance by reducing interference in a system with a severely limited capacity (Luck and Vogel, 1997; Hasher et al., 2008). Moreover, Vogel et al. (2005) used EEG to show that individuals with lower-memory capacity store more irrelevant information, suggesting that excessive encoding of distractions is associated with reduced memory capacity for relevant stimuli.

Beta desynchronization during the WM maintenance period was observed to be load dependent when the 4-item WM load condition is contrasted against the high-performing 2-item WM load trials. This is in agreement with previous findings that have linked alpha and beta desynchronization to an increased working memory load (Babiloni et al., 2004; Bocková et al., 2007; Hegner et al., 2007; Pesonen et al., 2007). Moreover, the current data supports the conclusion of Vogel et al. (2005) by showing that beta-band desynchronization during WM maintenance was comparable between 4 -item trials and low-performance 2-item trials, indicating irrelevant stimuli that were attended to were also encoded into WM stores. However, the data from Vogel et al. (2005) does not permit a dissection of independent selective attention processes for relevant and irrelevant information, since all stimuli were presented simultaneously. By dissociating neural signals from both to-be-attended and to-be-ignored stimuli, the current results revealed that effectively ignoring irrelevant information is the driving force behind optimal working memory performance. It further extends our understanding of this phenomenon by showing that a failure to ignore occurs very early in the visual processing stream. In addition, this study provides novel evidence that ineffective filtering of distractors impacts performance across participants, and is not just a characteristic of lowperforming individuals.

A deficit in inhibiting the processing of irrelevant information has been suggested as a source for the broad spectrum of cognitive deficits that occur in older adults (Hasher and Zacks, 1988). Functional magnetic resonance imaging data have supported this inhibitory deficit hypothesis of aging by showing that a top-down suppression deficit occurs in older adults and that the magnitude of the suppression deficit in visual processing areas correlates with WM performance impairment, such that reduced neural suppression of irrelevant information predicts decreased WM accuracy (Gazzaley et al., 2005a). In addition, recent EEG data has indicated that WM impairment in older adults is associated with a selective suppression deficit in ignoring irrelevant information early in the visual processing stream (Gazzaley et al., 2008). Here, we complement these findings by showing that even younger, healthy adults experience early processing suppression deficits on a trial-to-trial basis that negatively influences their subsequent WM performance.

Attentional modulation was found in posterior-occipital electrodes, consistent with the source localization of the P1 and N1 to the visual association cortex (Corbetta et al., 1990; Di Russo et al., 2003; Schoenfeld et al., 2007). The current study reveals that fluctuations in top-down modulation at very early stages of visual processing, specifically a failure to ignore distraction during the first $200 \mathrm{~ms}$ after stimulus onset, extends its influence into WM maintenance and results in diminished recognition performance, reflected by slower response times and reduced accuracy. This is not a claim that focusing attention on relevant information is inconsequential for WM, but that performance in young adults is not associated with fluctuations in focus on relevant stimuli, whereas variation in ignoring irrelevant information across trials influences WM accuracy and response time. These results support the assertion that attending and ignoring are not two-sides 
of the same coin, but are mechanistically dissociable processes. The current results reveal that a dependence on efficient filtering is critical for high-level performance in young adults, and provides an important neural link between attention and memory.

\section{References}

Awh E, Jonides J (2001) Overlapping mechanisms of attention and spatial working memory. Trends Cogn Sci 5:119-126.

Awh E, Vogel EK, Oh SH (2006) Interactions between attention and working memory. Neuroscience 139:201-208.

Babiloni C, Babiloni F, Carducci F, Cappa SF, Cincotti F, Del Percio C, Miniussi C, Vito Moretti D, Rossi S, Sosta K, Rossini PM (2004) Human cortical rhythms during visual delayed choice reaction time tasks - A high-resolution EEG study on normal aging. Behav Brain Res 153:261-271.

Beer AL, Röder B (2004) Attention to motion enhances processing of both visual and auditory stimuli: an event-related potential study. Cogn Brain Res 18:205-225.

Benjamini Y, Hochberg Y (1995) Controlling the false discovery rate - a practical and powerful approach to multiple testing. J R Stat Soc Ser B Stat Soc 57:289-300.

Bocková M, Chládek J, Jurák P, Halámek J, Rektor I (2007) Executive functions processed in the frontal and lateral temporal cortices: Intracerebral study. Clinical Neurophysiology 118:2625-2636.

Corbetta M, Shulman GL (2002) Control of goal-directed and stimulusdriven attention in the brain. Nat Rev Neurosci 3:201-215.

Corbetta M, Miezin FM, Dobmeyer S, Shulman GL, Petersen SE (1990) Attentional modulation of neural processing of shape, color, and velocity in humans. Science 248:1556-1559.

Cowan N (1995) Attention and memory: an integrated framework. New York: Oxford UP.

Delorme A, Makeig S (2004) EEGLAB: an open source toolbox for analysis of single-trial EEG dynamics including independent component analysis. J Neurosci Methods 134:9-21.

Di Russo F, Martínez A, Hillyard SA (2003) Source analysis of event-related cortical activity during visuo-spatial attention. Cereb Cortex 13:486-499.

Duncan J, Humphreys G, Ward R (1997) Competitive brain activity in visual attention. Curr Opin Neurobiol 7:255-261.

Eriksen BA, Eriksen CW (1974) Effects of noise letters upon identification of a target letter in a nonsearch task. Percept Psychophys 16:143-149.

Frith C (2001) A framework for studying the neural basis of attention. Neuropsychologia 39:1367-1371.

Fuster JM (1990) Inferotemporal units in selective visual attention and short-term memory. J Neurophysiol 64:681-697.

Gazzaley A, Cooney JW, Rissman J, D’Esposito M (2005a) Top-down suppression deficit underlies working memory impairment in normal aging. Nat Neurosci 8:1298-1300.

Gazzaley A, Cooney JW, McEvoy K, Knight RT, D’Esposito M (2005b) Topdown enhancement and suppression of the magnitude and speed of neural activity. J Cogn Neurosci 17:507-517.

Gazzaley A, Clapp W, Kelley J, McEvoy K, Knight RT, D’Esposito M (2008) Age-related top-down suppression deficit in the early stages of cortical visual memory processing. Proc Natl Acad Sci U S A 105:13122-13126.

Hardy JL, Delahunt PB, Okajima K, Werner JS (2005) Senescence of spatial chromatic contrast sensitivity. I. Detection under conditions controlling for optical factors. J Opt Soc Am A 22:49-59.

Hasher L, Zacks RT (1988) Working Memory, comprehension and aging: a review and a new view. In: The psychology of learning and motivation (Bower GH, ed), pp 193-225. New York: Academic.

Hasher L, Lustig C, Zacks JM (2008) Inhibitory mechanisms and the control of attention. In: Variation in working memory (Conway A, Kane M, Miyake A, Towse J, eds), pp 227-249. New York: Oxford UP.

Hillyard SA, Vogel EK, Luck SJ (1998) Sensory gain control (amplification) as a mechanism of selective attention: electrophysiological and neuroimaging evidence. Philos Trans R Soc Lond B Biol Sci 353:1257-1270.

LaBar KS, Gitelman DR, Parrish TB, Mesulam M (1999) Neuroanatomic overlap of working memory and spatial attention networks: a functional MRI comparison within subjects. Neuroimage 10:695-704.

Lavie N, Hirst A, de Fockert JW, Viding E (2004) Load theory of selective attention and cognitive control. J Exp Psychol Gen 133:339-354.

Li Hegner Y, Lutzenberger W, Leiberg S, Braun C (2007) The involvement of ipsilateral temporoparietal cortex in tactile pattern working memory as reflected in beta event-related desynchronization. Neuroimage 37:1362-1370.

Luck SJ, Vogel EK (1997) The capacity of visual working memory for features and conjunctions. Nature 390:279-281.

Mayer JS, Bittner RA, Nikolić D, Bledowski C, Goebel R, Linden DE (2007) Common neural substrates for visual working memory and attention. Neuroimage 36:441-453.

Pesonen M, Hämäläinen H, Krause CM (2007) Brain oscillatory 4-30 Hz responses during a visual $n$-back memory task with varying memory load. Brain Res 1138:171-177.

Ploner CJ, Ostendorf F, Brandt SA, Gaymard BM, Rivaud-Péchoux S, Ploner M, Villringer A, Pierrot-Deseilligny C (2001) Behavioural relevance modulates access to spatial working memory in humans. Eur J Neurosci 13:357-363.

Posner MI, Nissen MJ, Ogden WC (1978) Attended and unattended processing modes: the role of set for spatial location. In: Modes of perceiving and processing information (Pick HL, Saltzman IJ, eds). Hillsdale, NJ: Lawrence Erlbaum Associates.

Rainer G, Asaad WF, Miller EK (1998) Selective representation of relevant information by neurons in the primate prefrontal cortex. Nature 393:577-579.

Rugg MD, Milner AD, Lines CR, Phalp R (1987) Modulation of visual event-related potentials by spatial and nonspatial visual selective attention. Neuropsychologia 25:85-96.

Rypma B, D'Esposito M (2000) Isolating the neural mechanisms of agerelated changes in human working memory. Nat Neurosci 3:509-515.

Scerif G, Worden MS, Davidson M, Seiger L, Casey BJ (2006) Context modulates early stimulus processing when resolving stimulus-response conflict. J Cogn Neurosci 18:781-792.

Schoenfeld MA, Hopf JM, Martinez A, Mai HM, Sattler C, Gasde A, Heinze HJ, Hillyard SA (2007) Spatio-temporal analysis of feature-based attention. Cereb Cortex 17:2468-2477.

Sternberg S (1966) High-speed scanning in human memory. Science 153:652-654.

Sternberg S (1969) Memory-scanning: mental processes revaled by reaction-time experiments. Am Sci 57:421-457.

Stroop JR (1935) Studies of interference in serial verbal reactions. J Exp Psychol 18:643.

Tallon-Baudry C, Bertrand O, Peronnet F, Pernier J (1998) Induced $\gamma$-band activity during the delay of a visual short-term memory task in humans. J Neurosci 18:4244-4254.

Talsma D, Mulckhuyse M, Slagter HA, Theeuwes J (2007) Faster, more intense! The relation between electrophysiological reflections of attentional orienting, sensory gain control, and speed of responding. Brain Res 1178:92-105.

Valdes-Sosa M, Bobes MA, Rodriguez V, Pinilla T (1998) Switching attention without shifting the spotlight: object-based attentional modulation of brain potentials. J Cogn Neurosci 10:137-151.

Vogel EK, McCollough AW, Machizawa MG (2005) Neural measures reveal individual differences in controlling access to working memory. Nature 438:500-503.

Womelsdorf T, Fries P, Mitra PP, Desimone R (2006) Gamma-band synchronization in visual cortex predicts speed of change detection. Nature 439:733-736. 